\title{
Revisiting the differences between irreducible and reducible atlantoaxial dislocation in the era of direct posterior approach and C1-2 joint manipulation
}

\author{
Arsikere N. Deepak, MS, ${ }^{1}$ Pravin Salunke, MCh, ${ }^{1}$ Sushanta K. Sahoo, MCh, ${ }^{1}$ \\ Prashant K. Prasad, MS, ${ }^{1}$ and Niranjan K. Khandelwal, MD² \\ Departments of ${ }^{1}$ Neurosurgery and ${ }^{2}$ Radiodiagnosis, Postgraduate Institute of Medical Education and Research, Chandigarh,
India
}

\begin{abstract}
OBJECTIVE The current management of atlantoaxial dislocation (AAD) focuses on the C1-2 joints, commonly approached through a posterior route. The distinction between reducible AAD (RAAD) and irreducible AAD (IrAAD) seems to be less important in modern times. The roles of preoperative traction and dynamic radiographs are questionable. This study evaluated whether differentiating between the 2 groups is important in today's era.

METHODS Ninety-six consecutive patients with congenital AAD (33 RAAD and 63 IrAAD), who underwent surgery through a posterior approach alone, were studied. The preoperative and follow-up clinical statuses for both groups were studied and compared using Japanese Orthopaedic Association (JOA) scores. The radiological findings of the 2 groups were compared, and the intraoperative challenges described.
\end{abstract}

RESULTS A poor preoperative JOA score (clinical status) was seen in one-fifth of patients with IrAAD, although the mean JOA score was nearly similar in the RAAD and IrAAD groups. There was significant improvement in follow-up JOA score in both groups. However, segmentation defects (such as an assimilated arch of the atlas and C2-3 fusion) and anomalous vertebral arteries were found significantly more often in cases of IrAAD compared with those of RAAD. Os odontoideum was commonly seen in the RAAD group. The C1-2 joints were acute in IrAAD compared with RAAD. Preoperative traction in IrAAD resulted in vertical distraction and improvement in clinical and respiratory status. Surgery for IrAAD required much more drilling and manipulation of the $\mathrm{C1}-2$ joints while safeguarding the anomalous vertebral artery.

CONCLUSIONS Bony and vascular anomalies were much more common in patients with IrAAD, which made surgery more challenging than it was in RAAD despite similar approaches. An irreducible dislocation seen on preoperative radiographs made surgeons aware of difficulties that were likely to be encountered and helped them to better plan the surgery. Distraction achieved through preoperative traction reaffirmed the feasibility of intraoperative reduction. This made the differentiation between the 2 groups and the use of preoperative traction equally important.

https://thejns.org/doi/abs/10.3171/2016.8.SPINE16408

KEY WORDS atlantoaxial dislocation; basilar invagination; reducible; irreducible; C1-2 joints; anomalies; differences; cervical

$\mathrm{C}$ ONGENITAL atlantoaxial dislocation (AAD) is traditionally classified into irreducible or reducible. The dislocation is commonly seen in the anteroposterior plane (quantified by the atlantodental interval) or the vertical plane (defined as violation of the Chamberlain line, so-called basilar invagination [BI] Type I) ${ }^{4,5,8}$ Often it can be a combination of the 2 planes. Reducible AAD
(RAAD) is defined as C1-2 dislocation in either or both planes that reduces completely on extension or on application of traction. The failure to reduce completely despite traction is defined as irreducible AAD (IrAAD).

Until recently, the delineation between RAAD and IrAAD was important because the management of the 2 types differed..$^{11,12,14}$ IrAAD was treated with transoral de-

ABBREVIATIONS $A A D=$ atlantoaxial dislocation; $A D I=$ atlantodental interval $; \mathrm{BI}=$ basilar invagination; $C V J=$ craniovertebral junction; $\operatorname{IrAAD}=$ irreducible $\mathrm{AAD} ; \mathrm{JOA}=$ Japanese Orthopaedic Association; RAAD = reducible AAD; VA = vertebral artery; VD = vertical displacement.

SUBMITTED April 9, 2016. ACCEPTED August 17, 2016.

INCLUDE WHEN CITING Published online November 18, 2016; DOI: 10.3171/2016.8.SPINE16408. 
compression and posterior fusion, whereas RAAD could be treated by posterior fusion alone. ${ }^{14}$ This made it important to differentiate the two. The management of congenital AAD has significantly changed in the last 2 decades. Currently, the management for both IrAAD and RAAD focuses on opening and manipulating the $\mathrm{C} 1-2$ joints and fusing them through a direct posterior approach. 2,6,8,9,19,20,23 With the techniques involving joint reduction, the need to differentiate the two seems to be rapidly fading. Thus, the roles of dynamic radiography and preoperative traction are likely to be questioned.

The objective of this article is to highlight the clinical and radiological differences between IrAAD and RAAD that would determine the surgical planning even though the approach is the same. The intraoperative difficulties faced in each group are outlined. Additionally, we address the need to distinguish RAAD from IrAAD preoperatively and the role of preoperative traction from a contemporary perspective.

\section{Methods}

This study includes 96 consecutive patients with congenital AAD who underwent surgery at our institute between January 2013 and July 2015. Patients with a history of significant trauma, associated genetic syndromes (Down syndrome, Morquio syndrome, and achondroplasia), rheumatoid arthritis, and Chiari malformation were not included. The exclusion was necessary to reduce the heterogeneity of the cohorts.

Cases of BI Type 1 were included because they represent a true vertical or central dislocation of C-2 within C-1 (a type of AAD). This was irrespective of assimilation or nonassimilation of the atlas. The relationship between C-1 and C-2 is maintained in BI Type II, although it represents a significant craniovertebral anomaly., ${ }^{4,8}$ Therefore, patients with BI Type II were excluded from the study. A proper informed consent was obtained from all patients in addition to institutional ethical committee clearance.

\section{Clinical Radiological Assessment}

Patients' common clinical symptoms were spasticity and neck tilt. Clinical status was quantified using the Japanese Orthopaedic Association (JOA) score modified for an Indian setting. ${ }^{8}$ Breath-holding time was assessed in all patients. Radiological evaluation began with dynamic plain radiography of the craniovertebral junction (CVJ). The atlantodental interval (ADI) was calculated. Reconstructed thin-slice CT scans were obtained; $\mathrm{C} 1-2$ joint orientation and bony anomalies of the CVJ were studied. ${ }^{23}$ The vertical displacement (VD)/vertical dislocation (so-called BI Type I) was measured using the Chamberlain line as a reference on midsagittal CT scans. ${ }^{3}$ Bony anomalies noted were atlas assimilation, bifid atlas arch, os odontoideum, hypogenesis of odontoid, C2-3 or other cervical vertebral fusion, bifid vertebrae, and supernumerary joints. Using reconstructed images, inferior sagittal and coronal angles were measured as was VD. Sagittal angulation was measured between the inferior surface of $\mathrm{C}-1$ and the hard palate in the parasagittal plane ${ }^{23}$ Coronal obliquity was measured between the inferior surface of C-1 and plane of the foramen magnum as seen in the coronal plane..$^{23}$ Intensity changes at the CVJ and syrinx were noted on MRI. The size and course of the vertebral artery (VA) were noted on CT angiograms obtained in all patients. ${ }^{15,18,22}$ The presence of supernumerary facets or pseudojoints was noted. . $^{16,17}$

The dislocation was further classified into RAAD and IrAAD. A dynamic radiograph of the cervical spine was obtained. If $\mathrm{Cl}-2$ did not align on extension, cervical traction was applied. Crutchfield tongs were placed, and traction, starting with $7 \%-8 \%$ of body weight, was applied, with graded increases to a maximum of $7 \mathrm{~kg}$ over 24-48 hours. Lateral cervical radiographs were obtained to monitor the extent of reduction. RAAD was defined as complete C1-2 alignment on extension or application of cervical traction in both the vertical and anteroposterior planes. If the dislocation did not reduce despite cervical traction, it was labeled as an IrAAD. Partial reduction in any plane did not qualify as RAAD. Based on this criterion, 30 patients showed reduction on extension, and 3 patients showed complete $\mathrm{C} 1-2$ alignment after application of traction. Thus 33 patients had RAAD, and 63 patients had IrAAD.

\section{Preoperative Traction}

For patients who showed alignment on extension, the traction was applied just before the surgery. Those who did not show reduction on extension were subjected to skeletal traction for 48 hours prior to surgery. Lateral radiographs were obtained to monitor reduction as well as distraction. The JOA score and breath-holding time were assessed after 24 hours of traction in these patients.

\section{Surgical Technique}

The patients underwent surgery in the prone position. The C-2 lamina was traced to the C-2 facet. Bilateral C-2 root ganglia were transected to obtain a panoramic view of the $\mathrm{Cl}-2$ joints. The anomalous VA was addressed appropriately, by dissecting and safeguarding it. ${ }^{15,18,22}$ The artery was protected while drilling the $\mathrm{C} 1-2$ joints and inserting the screws.

The $\mathrm{C} 1-2$ joints were comprehensively drilled to make them flat or near normal in both sagittal and coronal planes. ${ }^{20}$ Metallic spacers packed with bone were used to compensate for bone loss after drilling and for VD. The spacers are hollow cuboids made of titanium, measuring $16 \mathrm{~mm}$ in length and $9 \mathrm{~mm}$ in width, with a graft window of $60 \%$. The struts connecting the superior and inferior surfaces measured $2 \mathrm{~mm}$ in width. Spacers are available in various heights from 4 to $9 \mathrm{~mm}$. The height of the spacer was chosen depending on the degree of vertical dislocation and the height of wedge of facet bone drilled. Bone grafts were obtained from the eighth or ninth rib. If joints were normal, minimal drilling was done only to make surfaces raw.

The posterior surface of the C-1 lateral mass beneath the C-1 arch was exposed completely. The midpoint of this facet surface was chosen as the insertion point for the $\mathrm{C}-1$ facet screw. A notch in the inferior surface of the C-1 arch (free or assimilated) was drilled to accommodate the tulip of the polyaxial screw. A polyaxial screw of $3.5 \mathrm{~mm}$ in di- 
TABLE 1. Distribution of patients with IrAAD and RAAD according to age and preoperative JOA score

\begin{tabular}{|c|c|c|c|c|c|c|}
\hline \multirow{2}{*}{$\begin{array}{l}\text { Preop } \\
\text { JOA Score }\end{array}$} & \multicolumn{3}{|c|}{ IrAAD (no. of patients [\%]) } & \multicolumn{3}{|c|}{ RAAD (no. of patients [\%]) } \\
\hline & $\leq 18$ Yrs & $19-40$ Yrs & $\geq 40 \mathrm{Yrs}$ & $\leq 18$ Yrs & $19-40$ Yrs & $\geq 40 \mathrm{Yrs}$ \\
\hline$\leq 7$ & $5(7.94)$ & $4(6.34)$ & $3(4.76)$ & $0(0.0)$ & $0(0.0)$ & $0(0.0)$ \\
\hline $8-13$ & $17(26.98)$ & $19(30.15)$ & $7(11.11)$ & $11(33.33)$ & $9(27.27)$ & $8(24.24)$ \\
\hline$\geq 14$ & $4(6.34)$ & $3(4.76)$ & $1(1.58)$ & $1(3.03)$ & $3(9.09)$ & $1(3.03)$ \\
\hline
\end{tabular}

ameter and $18-22 \mathrm{~mm}$ in length was inserted, $15^{\circ}$ medial and $15^{\circ}$ cranial, until the tulip was approximately $1 \mathrm{~mm}$ from the posterior surface of the lateral mass. This was irrespective of the assimilated or free status of the $\mathrm{C}-1$ arch. In cases of assimilated atlas, it was the lateral mass of C-1 assimilated with occipital condyle.

The C-2 isthmus was exposed. The medial margin of foramen transversarium was exposed. The superior-medial quadrant of the C-2 (medial-to-foramen transversarium) was prepared for screw insertion. A polyaxial screw of similar dimensions as that used for $\mathrm{C}-1$ was inserted approximately $10^{\circ}$ cranially and $30^{\circ}-40^{\circ}$ medially.

Rods were loosely fastened with screws and held with a long and stout rod holder; this rod holder acted as a lever and was manipulated to achieve $\mathrm{C} 1-2$ joint realignment in all planes. ${ }^{19}$ The screws were then compressed to jam the spacers and the rod was fastened tightly with the tulips. The realignment was confirmed by noting the position of C1-2 facets, occipital protuberance, and C-2 spinous process.

\section{Postoperative Assessment}

Clinical and radiological evaluations were performed at regular intervals. Postoperatively, thin-slice CT scans with reconstruction views were obtained to define the position of the screws and the extent of reduction. JOA scores were assessed postoperatively and at 4-6 months follow-up. An earlier CT was obtained if the patient had new progressive symptoms following surgery.

Bony fusion between the $\mathrm{C} 1-2$ facets was evaluated (by a radiologist and the surgeon) in CT scans obtained 6 months after surgery.

Thin reconstructed and reformatted (sagittal and coronal) CT images were obtained to look for fusion maturation and bone growth. With thin CT images, the scatter effect from newer metallic spacers is not an obstacle. The entire construct was evaluated. Presence of bony trabeculae between the $\mathrm{C} 1-2$ facets, or lamina and arch on CT scan, without any gap in between (with no mobility on flexion-extension radiographs), was defined as fusion. In the presence of metallic spacers, particular attention was paid to the area lateral, anterior, and posterior to the spacers. Cystic lucencies around the implants or along endplates and linear defects within the bridging trabeculae suggested nonfusion and required a repeat scan after 6 months. A scan at 6 months also showed subsidence or construct failure.

\section{Results}

The clinical and radiological differences between RAAD and IrAAD are listed in Tables 1 and 2. IrAAD and RAAD comprised 63 (65.62\%) and 33 (34.37\%) patients, respectively.

\section{Clinical Presentation}

Ages of patients ranged from 4 to 56 years. The majority of patients were in the age group 10-40 years. In both groups, the distribution of patients across age groups was almost uniform. The mean age at presentation was 32.14 years for RAAD and 32.06 years for IrAAD. Those patients who presented after 40 years of age comprised $27 \%$ of RAAD and $17 \%$ of IrAAD. Severe disability (JOA score $\leq 7)$ was seen in $12(19.05 \%)$ of patients with IrAAD, whereas no patient with RAAD had severe disability (Table 1). The mean breath-holding time was 16 seconds. It was $<10$ seconds in all patients with severe disability.

\section{Radiological Abnormalities}

Bony anomalies were seen in 61 (96.82\%) patients with IrAAD compared with $21(63.64 \%)$ patients with RAAD (Table 2). The most common bony anomaly in IrAAD was atlas assimilation in $50(79.36 \%)$ cases, followed by 47 (74.6\%) cases of C2-3 fusion. In RAAD, os odontoideum in $16(48.48 \%)$ cases was the most common anomaly. Bifid or hemi-ring atlas partially assimilated was seen more often in IrAAD. A free bifid atlas was seen in RAAD. Supernumerary joints were present in 8 patients with IrAAD and in 2 with RAAD. The mean ADI was $7.25 \mathrm{~mm}$ and $6.49 \mathrm{~mm}$ for RAAD and IrAAD, respectively. The mean VD (before traction) was $0.94 \mathrm{~mm}$ for RAAD and 4.99 $\mathrm{mm}$ for IrAAD, and the difference was significant $\mathrm{p}<$ $0.05)$.

The atlas arch could be intact (Fig. 1) or assimilated (Fig. 2) in patients with RAAD. The atlas was often assimilated in IrAAD (Fig. 3). Preoperatively, the inferior sagittal angles were $138.93^{\circ} \pm 15^{\circ}$ in IrAAD and $156.57^{\circ} \pm 15^{\circ}$ in RAAD. The sagittal angles were significantly acute in patients with IrAAD compared with RAAD. Additionally, there was significant correlation between sagittal angle and age at presentation in IrAAD but not in RAAD. Age at presentation correlated with the degree of the inferior sagittal angle; the more acute the angle, the younger was the age at presentation in IrAAD. In all patients, postoperative inferior sagittal angle indices were corrected to near normal (Figs. 1 and 3-6).

Coronal angles were $141.96^{\circ} \pm 10^{\circ}$ in IrAAD and $149.03^{\circ} \pm 9^{\circ}$ in RAAD. The difference was not significant. However, at least 1 coronal angle in IrAAD was acute, and asymmetry was seen in almost all cases of IrAAD.

We observed a VA anomaly in $34(26.98 \%)$ sides in IrAAD compared with 5 (7.57\%) sides in RAAD (Fig. 7). Common VA anomalies were medial looping of VA in 8 
TABLE 2. Clinical and radiological differences between RAAD and IrAAD groups for each age group

\begin{tabular}{|c|c|c|c|c|c|c|}
\hline \multirow[b]{2}{*}{ Variable } & \multicolumn{3}{|c|}{ IrAAD } & \multicolumn{3}{|c|}{ RAAD } \\
\hline & $\leq 18$ Yrs & $19-40$ Yrs & $\geq 40$ Yrs & $\leq 18$ Yrs & $19-40$ Yrs & $\geq 40 \mathrm{Yrs}$ \\
\hline No. of patients & 26 & 26 & 11 & 12 & 12 & 9 \\
\hline \multicolumn{7}{|l|}{ Mean JOA score } \\
\hline Preop & 10.19 & 10.11 & 11 & 10.66 & 11.25 & 10.66 \\
\hline Postop & 11.61 & 11.76 & 12.45 & 12.58 & 11.91 & 12.55 \\
\hline Follow-up & 13.5 & 13.75 & 12.81 & 13.41 & 13.58 & 11.66 \\
\hline \multicolumn{7}{|l|}{ Mean extent of dislocation, $\mathrm{mm}$} \\
\hline Preop ADI & 6.66 & 6.92 & 5.9 & 7.25 & 7.5 & 7 \\
\hline Postop ADI & 2.07 & 2 & 1.63 & 1.75 & 1.83 & 2 \\
\hline Preop VD & 5.69 & 5.84 & 3.45 & 1.08 & 1.08 & 0.66 \\
\hline Postop VD & 0.34 & 0.65 & 0.63 & 0.16 & $0^{*}$ & $0^{*}$ \\
\hline \multicolumn{7}{|l|}{ C1-2 joint orientation $\left(^{\circ}\right)$} \\
\hline C-1 inferior facet sagittal angle & 128.8 & 134.2 & 143.8 & 156.91 & 151.83 & 160.88 \\
\hline Corrected inferior facet sagittal angle (postop) & 177.76 & 178.9 & 176.7 & 176.66 & 177.5 & 176.88 \\
\hline C-1 coronal inferior facetal angle & 136.4 & 142.84 & 144 & 149.5 & 154.5 & 148.11 \\
\hline Corrected coronal inferior facetal angle (postop) & 158.76 & 153 & 154.18 & 154.16 & 156.66 & 159.77 \\
\hline \multicolumn{7}{|l|}{ Anomalous VA (of 192 sides) } \\
\hline Persistent 1st intersegmental artery & 1 & 4 & 0 & 1 & 1 & 0 \\
\hline Fenestration of VA & 3 & 1 & 0 & 0 & 0 & 0 \\
\hline Anomalous PICA & 1 & 0 & 0 & 0 & 0 & 0 \\
\hline Medial loop of VA & 3 & 2 & 1 & 0 & 1 & 1 \\
\hline Inverted VA & 3 & 3 & 0 & 0 & 0 & 0 \\
\hline Atrophic & 3 & 6 & 3 & 0 & 1 & 0 \\
\hline \multicolumn{7}{|l|}{ Associated bony anomalies, no. of patients } \\
\hline Atlas assimilation & 20 & 23 & 7 & 1 & 3 & 2 \\
\hline Bifid atlas/hemi-ring atlas & 5 & 10 & 1 & 2 & 2 & 0 \\
\hline Os odontoideum & 3 & 5 & 1 & 7 & 6 & 3 \\
\hline C2-3 fusion & 19 & 20 & 8 & 0 & 2 & 1 \\
\hline Pseudofacet & 0 & 8 & 0 & 0 & 2 & 0 \\
\hline
\end{tabular}

(4.17\%) cases, followed by 7 (3.64\%) cases of first intersegmental artery type of VA.

\section{Results of Traction}

Traction improved spasticity and pulmonary function in most of the patients with IrAAD. There was subjective improvement in spasticity in most patients, although JOA scores improved by only 1 point in 39 (61.9\%) patients. On traction, the JOA score could not be assessed properly because the patients were immobilized and those with preoperative bladder involvement were catheterized. The breath-holding time improved by 5 seconds in 10 of 13 patients with severe disability. The vertical distraction (partial reduction of $\mathrm{AAD}$ in the vertical plane but not in the anteroposterior plane) was seen on post-traction radiographs in 40 patients with IrAAD. However, it was difficult to quantify VD on radiographs. Post-traction CT scans were not obtained

\section{Results of Surgery}

The $\mathrm{C} 1-2$ joints were opened in all patients. Metallic spacers were used in all patients with IrAAD and in 11 (33\%) patients with RAAD to compensate for bone loss due to drilling.

The surgery for IrAAD was more challenging. It was important to mobilize the $\mathrm{C} 1-2$ joint or to convert the IrAAD into RAAD before fusion. This was an additional, relatively difficult step that was not required in cases of RAAD. The difficulty level in IrAAD increased with the presence of pseudofacets, an anomalous VA, os odontoideum, or C1-2 joints with very acute sagittal and coronal inclination (Fig. 6). Os odontoideum was seen in 16 $(48.48 \%)$ patients, with RAAD. There was no added difficulty during surgery. However, 9 (14.28\%) patients with IrAAD had os odontoideum. In these cases, the facets were dislocated significantly and required more manipulation to reduce.

\section{Intraoperative Complications}

Intraoperative complications occurred in 2 patients with IrAAD and 1 patient with RAAD. Surgery was uneventful in $96.8 \%$ of cases. One patient with IrAAD had in- 

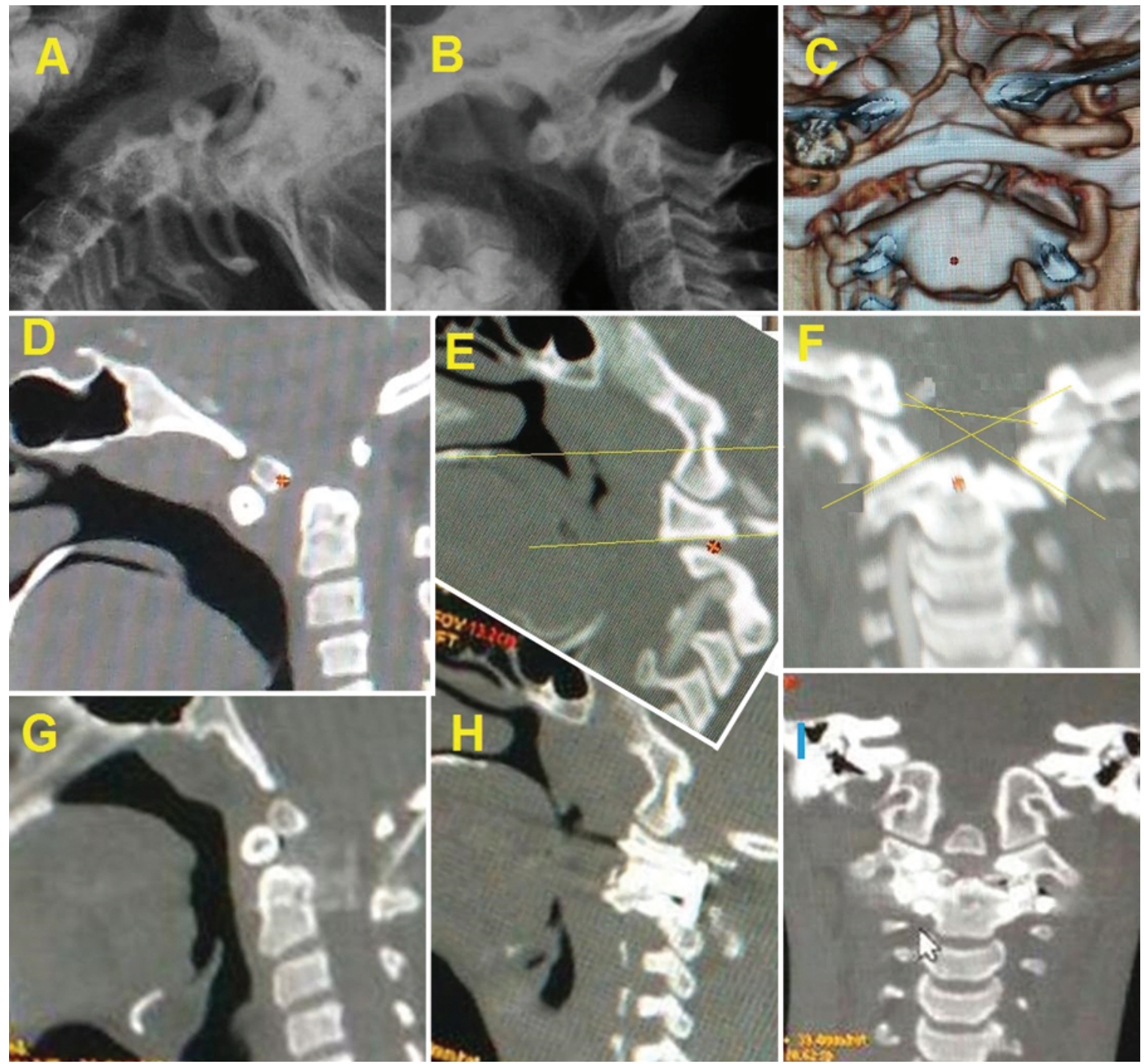

FIG. 1. Images obtained in a patient with RAAD with os odontoideum. A and B: Dynamic radiographs showing reduction of $A A D$ on extension. C: 3D-reconstructed CT image and angiogram of CVJ showing normal course of the VA and normal joints. D: Midsagittal CT image showing os odontoideum with AAD. E: Parasagittal CT image showing normal C1-2 joint with normal C-1 sagittal angulation $\left(175^{\circ}\right)$ in relation to hard palate. F: A coronal CT image showing symmetrical $\mathrm{C} 1-2$ joints with normal coronal inclination $\left(150^{\circ}\right)$. G-I: Corresponding postoperative CT images showing fusion of C1-2 joints in reduced position (arrow). Figure is available in color online only.

traoperative, severe bradycardia during manipulation. He awoke with quadriplegia and no spontaneous respiration, suggestive of intraoperative spinal cord injury. Anomalous VAs were injured in 2 patients (bilaterally in 1 patient with IrAAD and the dominant VA in 1 patient with RAAD) and were repaired. However, all 3 of these patients had eventful postoperative courses and died. Posterior circulation infarcts were seen in the 2 patients with VA injury.

\section{Surgical Outcomes}

Paired-samples correlation showed that JOA scores improved during the follow-up period compared with the immediate postoperative and preoperative periods; the difference was significant $(\mathrm{p}<0.01)$ (Table 3). The mean follow-up was 18.4 months (9-36 months) for IrAAD and 18.8 months (10-38 months) for RAAD. The mean JOA score at follow-up was 13.47 and 13.42 for IrAAD and RAAD, respectively (most patients resumed work and were independent). Clinical outcomes at 3-month followup showed improvement in $95.83 \%$ of patients across both groups. A follow-up JOA score $\geq 14$ was seen in 53.96\% of patients with IrAAD and in $54.54 \%$ of patients with RAAD. There was a significant change in ADI $(p<0.05)$ postoperatively compared with preoperative measurement in both groups. Intraoperative reduction and alignment were achieved in all cases. No patient required transoral odontoidectomy during the postprocedure period.

All patients had good fusion 6 months after primary or repeat surgery. The mean follow-up was 19 months.

\section{Postoperative Period and Complications}

Among patients, $19.3 \%$ had wound infections, which 


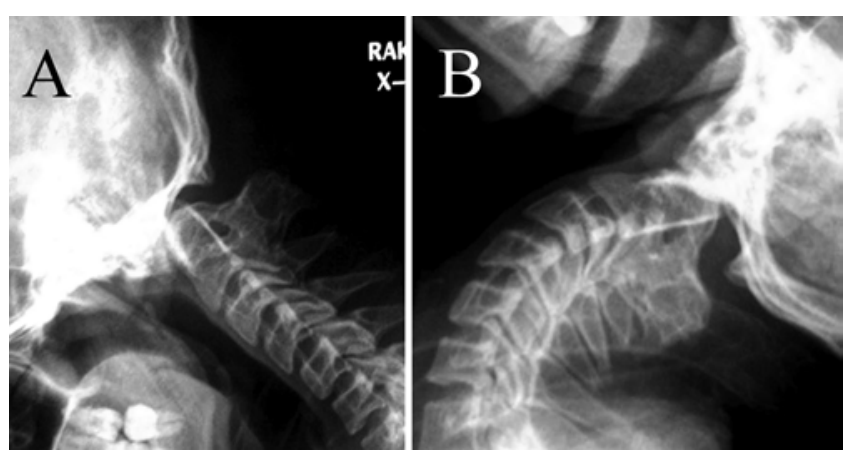

FIG. 2. Radiographs obtained in a patient with RAAD with assimilated arch of the atlas. A: Radiograph in flexion showing AAD. B: Radiograph in extension showing adequate reduction. Note the assimilated atlantal arch and C2-3 fusion.

were treated appropriately. Two patients had major complications, and during the postoperative period one patient had respiratory distress that required prolonged ventilation but later improved. One patient presented 8 weeks after surgery with neck pain and tilt. This patient had slippage of the spacer on the right side, causing VD and lateral tilt, which was corrected by repeat surgery with further remodeling of the joint and repositioning of the larger spacer. Good fusion was seen in this patient 6 months after the second surgery.

Three patients ( 2 with IrAAD and 1 with RAAD) died during the perioperative period. These patients had intraoperative complications as described above. Another patient with IrAAD, who had a good immediate postoperative outcome, did not return for follow-up. It was learned that the patient died (approximately 3 months after surgery), without any obvious cause.

\section{Discussion}

\section{Clinical Presentation and Age at Presentation}

Progressive spastic quadriparesis was the most common presentation in both groups at all ages. A poor-grade JOA score (severe disability) was more often seen with IrAAD. Neck tilt was more common in IrAAD, especially among patients with lateral angular dislocation. The majority of patients, with either RAAD or IrAAD, were younger than 40 years at presentation.

\section{Role of Preoperative Traction}

Preoperative traction played a major role approximately a decade ago, because reducibility on traction changed the management completely. In today's era, when the majority of surgeons prefer joint manipulation and intraoperative reduction through a posterior route, the role of traction is debatable. We still prefer to apply preoperative traction in IrAAD for the following reasons. First, most of the patients did seem to show improvement in clinical status, especially spasticity and respiration, after application of traction. Second, vertical distraction was seen in most of the patients with IrAAD, the majority of whom had a component of vertical dislocation (so-called BI). This distraction suggests the feasibility of $\mathrm{C} 1-2$ joint manipulation and intraoperative reduction. If distraction is not seen on post-traction radiographs, one can preempt a greater difficulty in intraoperative reduction. Third, although theoretical, traction is likely to break fibrotic ligaments and the joint capsule in these cases with long-standing irreducibility. This makes it easy to open the joint space, which is critical, especially in the presence of anomalous VAs or pseudofacets.

\section{Radiological Differences}

Segmentation abnormalities, such as assimilated C-1 and $\mathrm{C} 2-3$ fusion, were significantly higher in cases of IrAAD. A bifid atlas with assimilation was seen with irreducible lateral angular AAD. ${ }^{21}$ However, a bifid-free atlas and os odontoideum were often seen with RAAD. The incidence of anomalous VAs was $26.9 \%$ in IrAAD compared with $5.75 \%$ in RAAD, and the difference was statistically significant $(\mathrm{p}<0.01)$. Similarly, pseudofacets were more often seen in patients with IrAAD (12.69\%) compared with RAAD (6.06\%). ${ }^{15-18,22}$ This clearly suggests that the abnormality of the entire sclerotome affects all structures developing from it.
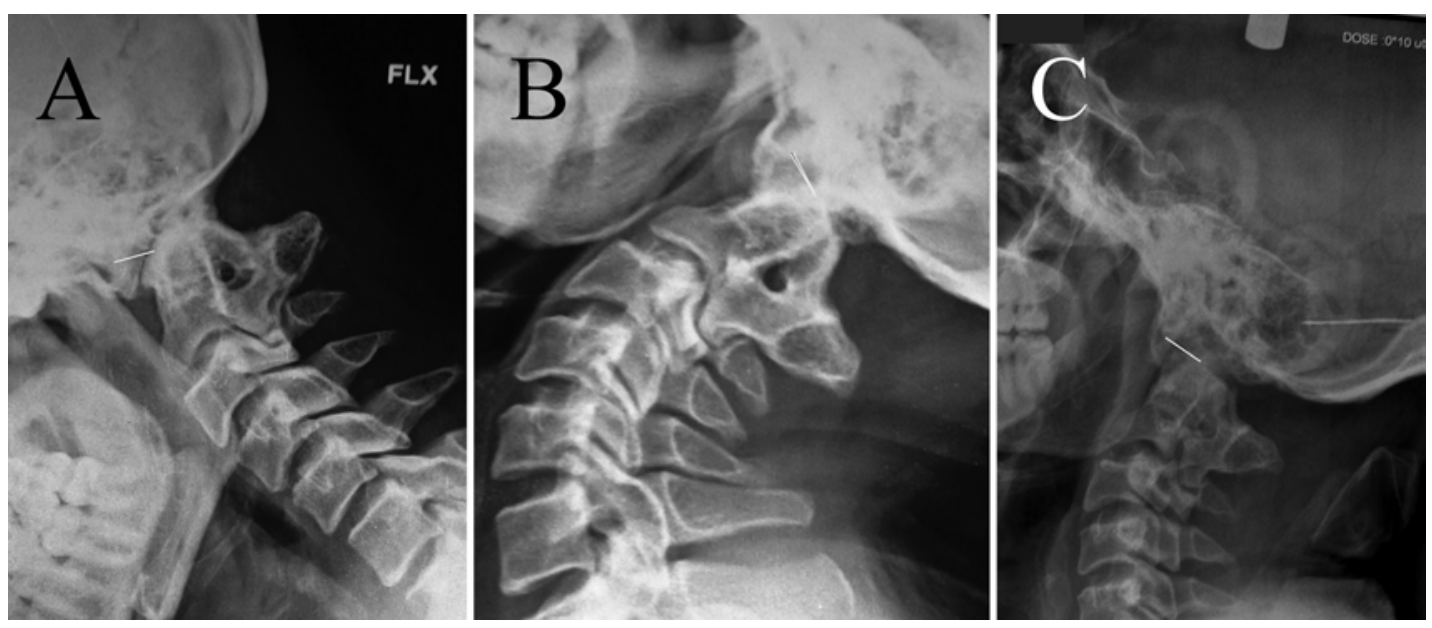

FIG. 3. Radiographs obtained in a patient with IrAAD with assimilated arch of the atlas: in flexion showing AAD (A), in extension showing no reduction (note the assimilated atlas arch and C2-3 fusion) (B), and post-traction showing incomplete reduction (C). 


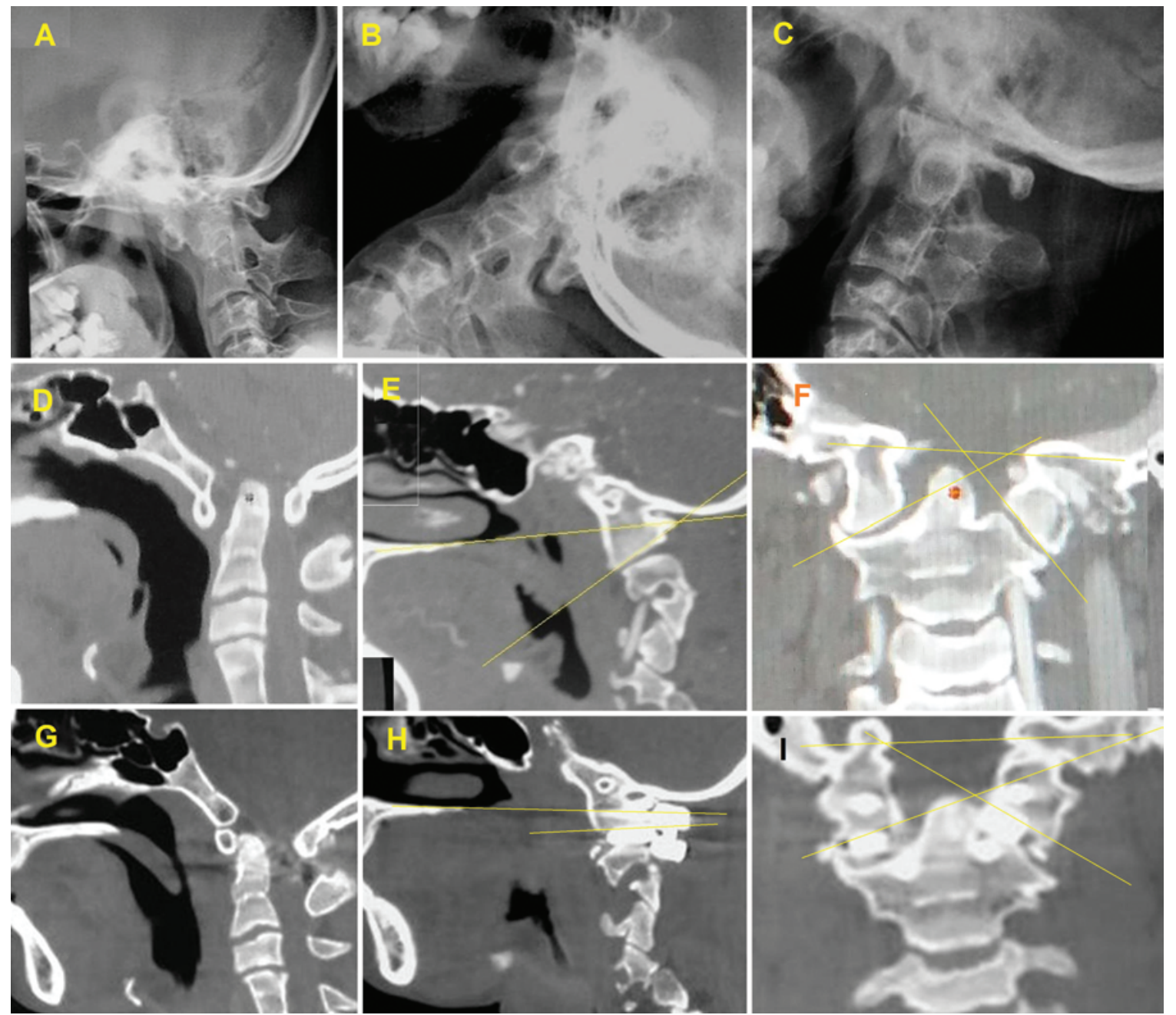

FIG. 4. Images obtained in a patient with IrAAD. A and B: Dynamic radiographs showing no reduction of AAD (IrAAD) on extension. Note the assimilated arch of the atlas and C2-3 fusion. C: Post-traction radiograph demonstrating distraction but no anteroposterior reduction. D: Midsagittal CT image showing vertical (BI) and anteroposterior AAD. E: Parasagittal CT image showing acute angulation of $\mathrm{C}-1$ facet in relation to hard palate $\left(137^{\circ}\right.$; yellow lines). F: Coronal CT image showing asymmetrical C1-2 joints with left inferior $\mathrm{C}-1$ facet coronal angle of $135^{\circ}$ and a right side angle of $150^{\circ}$ (yellow lines) with reference to the foramen magnum edge. G: Postoperative midsagittal CT image showing reduced dislocation. H: Parasagittal CT image showing the flattened C1-2 joint (yellow lines). I: Drilled medial aspect of left C1-2 joint in coronal section to flatten it (yellow lines). Figure is available in color online only.

In patients with IrAAD, the $\mathrm{C} 1-2$ joints were oblique, both in sagittal and coronal planes. The joint orientation can be quantified using technique described by Chandra et al. ${ }^{1}$ or Salunke et al. ${ }^{23}$ We chose the latter method. The joints were relatively normal or slightly oblique in patients with RAAD. Additionally, the obliquity of joints correlated well with the age at presentation in patients with IrAAD (Fig. 5). There was no such correlation found in patients with RAAD. This clearly suggests that facet orientation plays a major role in cases of IrAAD.

These patients were possibly born with deformed oblique joints but not dislocated joints. The obliquity of the joints determines the slippage rate of $\mathrm{C}-1$ over $\mathrm{C}-2$ in various planes. To begin with, they may reduce on extension. A sagittally oblique joint would slip more anteriorly, whereas a vertical coronal inclination would lead to central or vertical dislocation. This vertical dislocation is the same as traditional BI Type I. ${ }^{10}$ Usually, it is a combination of vertical and anteroposterior dislocation (mixed). ${ }^{10}$ The more oblique joint would slip faster, produce cord compression, and become symptomatic earlier compared with those with less oblique joints. ${ }^{23}$ With aging, there would be additional compromise because of degenerative changes. These degenerative changes are obviously more prevalent in cases with increased stress due to an assimilated atlas and $\mathrm{C} 2-3$ fusion.

Ligaments and soft tissues are the probable cause of dislocation in RAAD, although the facets are slightly oblique. The bony anomalies, such as os odontoideum, are seen in patients of all ages. It clearly suggests that incompetence of ligaments plays a major role in patients with RAAD. The reason for variability in age at presentation remains speculative. It is possible that these patients have weak ligaments. Those with major ligamentous incom- 

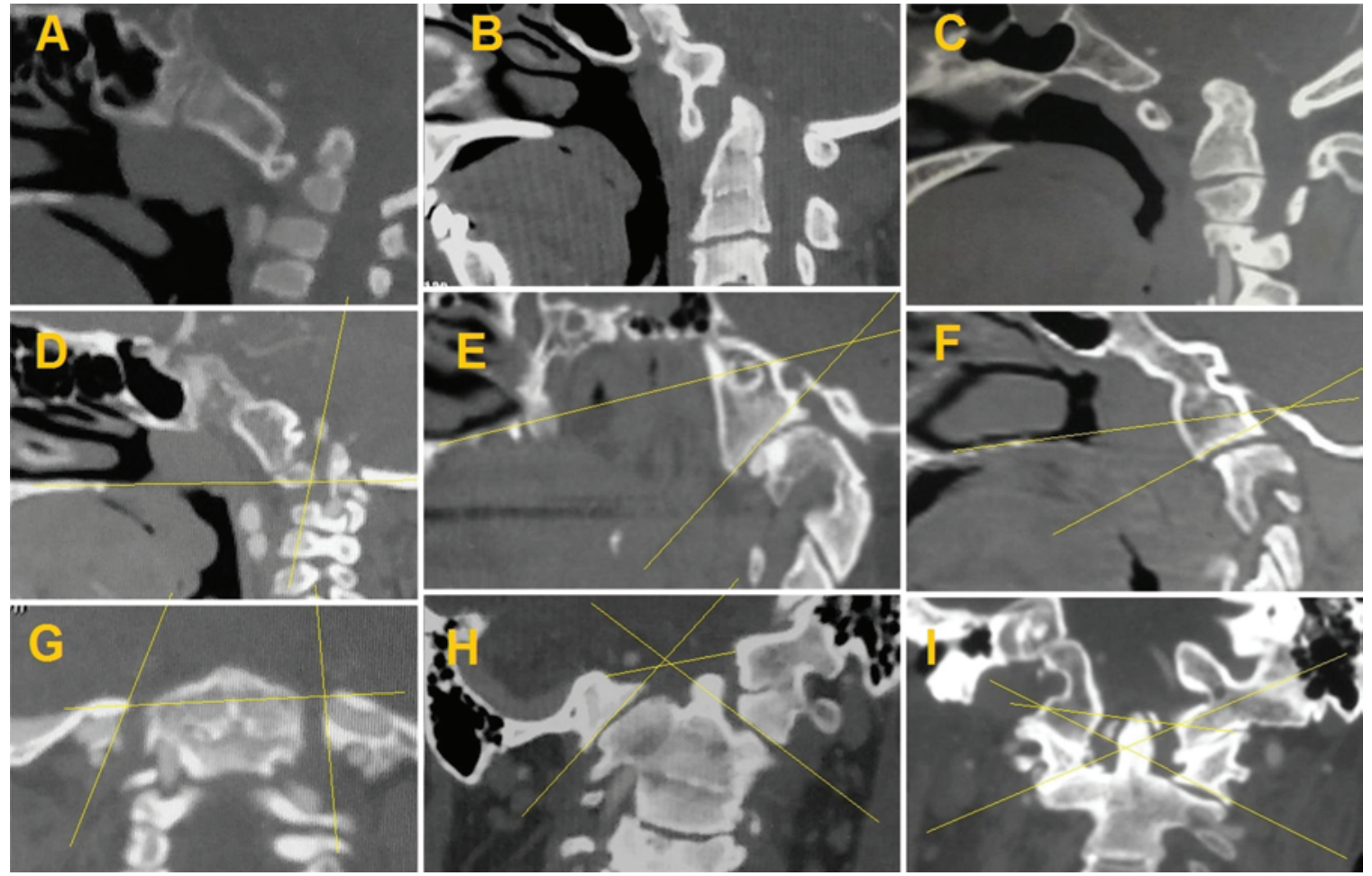

FIG. 5. CT images obtained in patients presenting at different ages with IrAAD with VD (BI Type I). Midsagittal CT images of a 4-year-old (A), 25-year-old (B), and 52-year-old (C) patient with IrAAD and BI (vertical dislocation). The ADI is almost the same. The corresponding parasagittal CT images show the acute C1-2 joints (yellow lines). The joint is extremely acute or vertical in the 4-year-old (D), slightly less acute $\left(132^{\circ}\right)$ in the 25 -year-old $(E)$, and even less acute $\left(147^{\circ}\right)$ in the 52 -year-old $(F)$ patient. Similarly, the coronal angulation is extremely acute (almost vertical) in the 4-year-old $(\mathbf{G})$, slightly less oblique in the 25 -year-old $(\mathbf{H})$, and almost normal in the 52-year-old (I) patient. Figure is available in color online only.

petence would present earlier. Those with relatively better ligaments would present later. With age, degenerative changes add to the stress on a relatively weak ligament, giving rise to an increase in dislocation and producing symptoms.

Another observation was that patients with IrAAD, who had os odontoideum but did not exhibit a reduction on traction, had relatively fewer oblique joints. This suggests that there was severe spondyloptosis in these cases, causing irreducibility. Intraoperatively, these cases required greater manipulation to achieve complete reduction (Fig. 6).

\section{Intraoperative Findings}

It is obvious that operative difficulties were more often encountered in patients with IrAAD compared with RAAD. Facet drilling was increased in cases of IrAAD because the joints were more oblique. Comprehensive drilling of facets or facet osteotomy leads to loss of bone and requires compensation with metallic spacers. Metallic spacers were used in $33 \%$ of patients with RAAD and in all patients with IrAAD, although the majority of patients with RAAD had minimal VD. The advantage of using a spacer is that it avoids complications caused by bone-graft subsidence due to possible resorption, hence leading to repeated dislocation.

The VA was often anomalous, requiring it to be dissected and safeguarded. ${ }^{15,18,22}$ Manipulation of $\mathrm{C} 1-2$ joints to achieve realignment was required in IrAAD. Manipu- lation is extremely important, especially in patients with lateral angular AAD. Certain cases of IrAAD, especially those with os odontoideum, required a combination of force and delicate maneuvering to achieve good reduction without injuring the spinal cord. This was possibly because the entire C-1 facet had slipped anteriorly onto C-2. Such a locked facet caused irreducibility and made the surgery more difficult (Fig. 6). These steps require experience, making surgery for IrAAD more challenging than RAAD.

\section{Outcomes of Patients With RAAD Versus IrAAD}

In this series, we did not notice any significant difference in the outcomes of patients with RAAD versus IrAAD. Irrespective of the type of AAD, outcomes were uniformly good. However, some patients with IrAAD died. The cause of death in 1 patient with IrAAD during follow-up in remains speculative. It may have been due to slippage of spacers.

In a previous series, we observed that nonambulatory patients with IrAAD fared better than those with RAAD at follow-up. ${ }^{14}$ The reason speculated was multiple injuries to the cord. Because there were no patients with RAAD with a poor preoperative grade in the current series, it is difficult to comment.

Finally, an artificial $\mathrm{Cl}-2$ joint would be more feasible in RAAD than IrAAD, making it important to differentiate between the 2 varieties. ${ }^{13}$ 

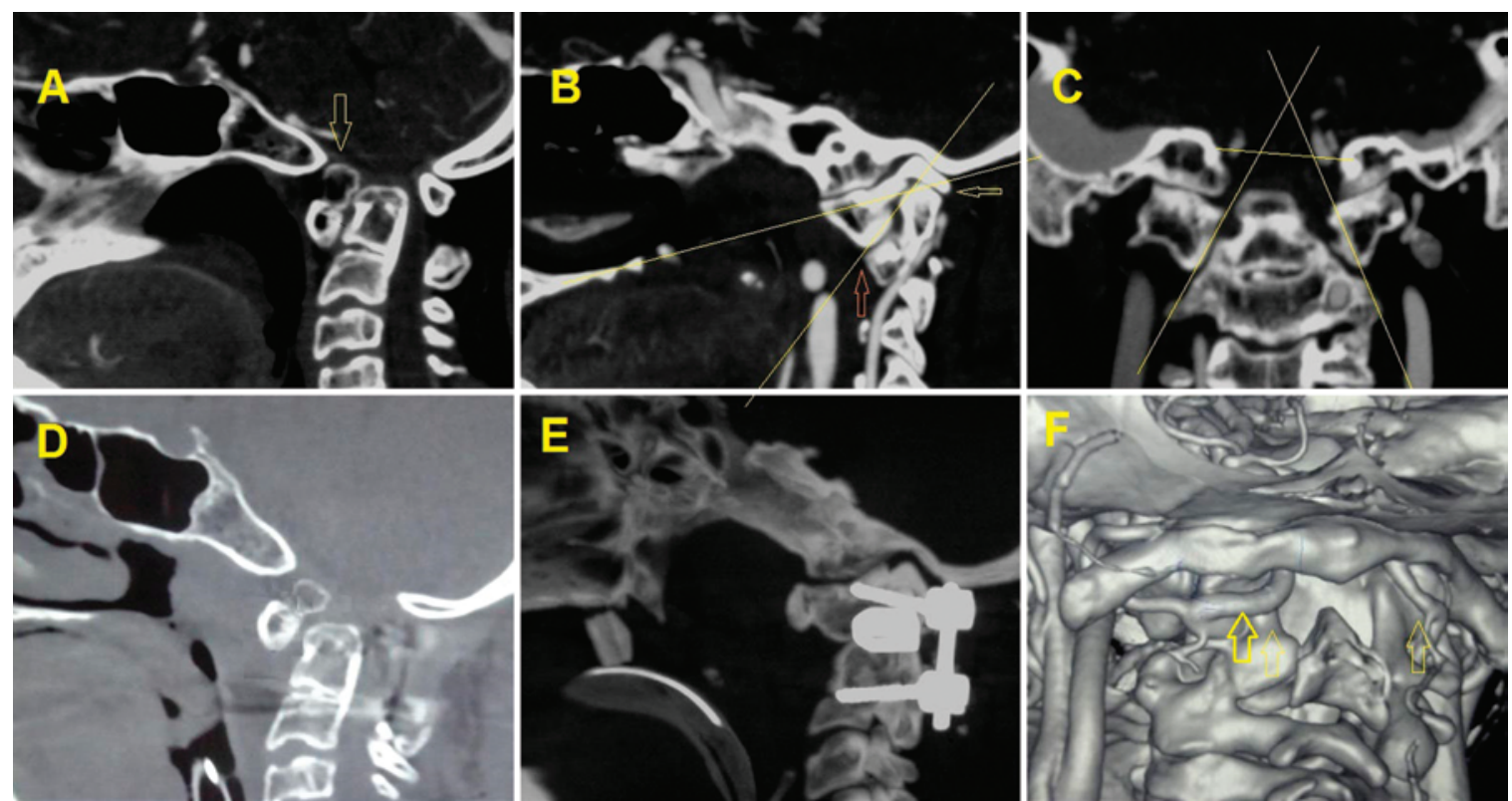

FIG. 6. CT scans obtained in patients with IrAAD that posed various surgical challenges. A: Midsagittal image obtained in a patient with IrAAD and BI with os odontoid (arrow) and intact C-1 arch. B: Parasagittal cut showing acute C1-2 joint (anterior arrow) and pseudofacet (posterior arrow). C: The coronal angulation of bilateral C1-2 joint is acute (yellow lines). D: Postoperative midsagittal image showing reduction. E: Postoperative parasagittal image showing flattened C1-2 joint with spacer and screws. F: Preoperative CT angiogram showing the VA crossing the joint posteriorly (arrows), making the surgery difficult. Figure is available in color online only.

The higher rate of wound infections in our series was probably due to increased sweating within the hard cervical collar and poor hygiene, compounded by decreased sensations in the suboccipital area due to cutting of C-2 nerve root ganglion. To prevent this, the collar was changed at regular intervals, and the wound was cleaned at least twice per day.

\section{Limitations of the Study}

The role of traction was not objectively assessed. The JOA score is not appropriate for patients on traction because they are immobilized, and the improvement in ambulation cannot be assessed. Similarly, the urinary catheter was not removed after application of traction, and improvement in bladder function was not assessed. The post-traction vertical reduction was not assessed objectively because post-traction CT scans were not obtained.

Breath-holding time is a crude method to assess pulmonary function. The pre- and post-traction assessment using breath-holding time gives only a rough estimate. Finally, the follow-up was not long enough to comment on the growth impairment in children.

\section{Conclusions}

The incidence of segmentation defects was significantly higher in IrAAD, whereas os odontoideum was seen significantly more often in RAAD. Similarly, an anomalous VA was often seen with IrAAD. The joints were oblique in both the sagittal and coronal planes in IrAAD. In today's era, when both varieties of AAD are approached through
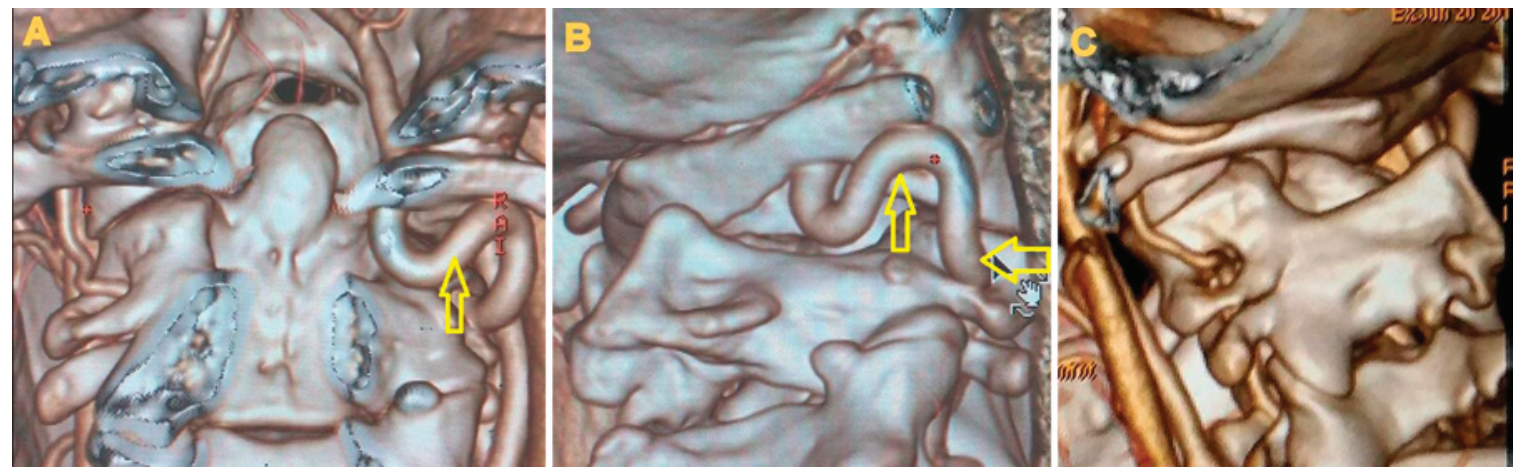

FIG. 7. A: 3D reconstructed CT image (posterior view) showing normal course of the left VA and anomalous right VA (arrow). B: Oblique view showing the right C1-2 joint. The VA is crossing the C1-2 joint posteriorly (arrows). C: Oblique view showing the left C1-2 joint. The VA has a normal course and is lateral to the joint. Figure is available in color online only. 
TABLE 3. Preoperative, postoperative, and follow-up JOA scores in RAAD and IrAAD

\begin{tabular}{|c|c|c|c|c|c|}
\hline \multirow[b]{2}{*}{ Group } & \multirow[b]{2}{*}{$\begin{array}{l}\text { JOA } \\
\text { Score }\end{array}$} & \multicolumn{3}{|c|}{ No. of Patients (\%) } & \multirow{2}{*}{$\begin{array}{l}\text { Mean Follow- } \\
\text { Up for Each } \\
\text { Group (mos) }\end{array}$} \\
\hline & & Preop & Postop & $\begin{array}{c}\text { 6-Mo } \\
\text { Follow-Up }\end{array}$ & \\
\hline \multicolumn{6}{|l|}{ IrAAD } \\
\hline & $\leq 7$ & $12(19.00)$ & $0(0.00)$ & $0(0.00)$ & 19.9 \\
\hline & $8-13$ & $43(68.30)$ & $40(63.49)$ & $26(41.26)$ & 19.3 \\
\hline & $\geq 14$ & $8(12.70)$ & $21(33.33)$ & $34(53.96)$ & 16 \\
\hline \multicolumn{6}{|l|}{ RAAD } \\
\hline & $\leq 7$ & $0(0.00)$ & $0(0.00)$ & $0(0.00)$ & - \\
\hline & $8-13$ & $28(84.80)$ & $23(69.69)$ & $14(42.40)$ & 19.1 \\
\hline & $\geq 14$ & $5(15.20)$ & $10(30.30)$ & $18(54.54)$ & 18.5 \\
\hline
\end{tabular}

$-=$ not applicable.

the posterior route, associated abnormalities make surgery for IrAAD more challenging than for RAAD. Differentiating RAAD from IrAAD helps the surgeon preempt the likely intraoperative challenges and plan accordingly. Preoperative traction results in distraction or partial reduction of IrAAD, with improvements in spasticity and respiratory status, and reassures the surgeon of the feasibility of intraoperative reduction.

\section{References}

1. Chandra PS, Goyal N, Chauhan A, Ansari A, Sharma BS, Garg A: The severity of basilar invagination and atlantoaxial dislocation correlates with sagittal joint inclination, coronal joint inclination, and craniocervical tilt: a description of new indexes for the craniovertebral junction. Neurosurgery 10 (Suppl 4):621-630, 2014

2. Chandra PS, Prabhu M, Goyal N, Garg A, Chauhan A, Sharma BS: Distraction, compression, extension, and reduction combined with joint remodeling and extra-articular distraction: description of 2 new modifications for its application in basilar invagination and atlantoaxial dislocation: prospective study in 79 cases. Neurosurgery 77:67-80, 2015

3. Cronin CG, Lohan DG, Mhuircheartigh JN, Meehan CP, Murphy J, Roche C: CT evaluation of Chamberlain's, McGregor's, and McRae's skull-base lines. Clin Radiol 64:64-69, 2009

4. Ferreira ED, Botelho RV: Atlas assimilation patterns in different types of adult craniocervical junction malformations. Spine (Phila Pa 1976) 40:1763-1768, 2015

5. Ferreira JA, Botelho RV: The odontoid process invagination in normal subjects, Chiari malformation and basilar invagination patients: Pathophysiologic correlations with angular craniometry. Surg Neurol Int 6:118, 2015

6. Goel A: Treatment of basilar invagination by atlantoaxial joint distraction and direct lateral mass fixation. J Neurosurg Spine 1:281-286, 2004

7. Goel A, Bhatjiwale M, Desai K: Basilar invagination: a study based on 190 surgically treated patients. J Neurosurg 88:962-968, 1998

8. Goel A, Kulkarni AG, Sharma P: Reduction of fixed atlantoaxial dislocation in 24 cases: technical note. J Neurosurg Spine 2:505-509, 2005

9. Goel A, Shah A: Atlantoaxial facet locking: treatment by facet manipulation and fixation. Experience in 14 cases. J Neurosurg Spine 14:3-9, 2011

10. Jain VK: Atlantoaxial dislocation. Neurol India 60:9-17, 2012
11. Jain VK, Behari S: Management of congenital atlanto-axial dislocation: some lessons learnt. Neurol India 50:386-397, 2002

12. Menezes AH: Craniovertebral junction database analysis: incidence, classification, presentation, and treatment algorithms. Childs Nerv Syst 24:1101-1108, 2008

13. Salunke P: Artificial atlanto-axial joints: On the "move". Neurol India 64:275-278, 2016

14. Salunke P, Behari S, Kirankumar MV, Sharma MS, Jaiswal AK, Jain VK: Pediatric congenital atlantoaxial dislocation: differences between the irreducible and reducible varieties. J Neurosurg 104 (2 Suppl):115-122, 2006

15. Salunke P, Futane S, Sahoo SK, Ghuman MS, Khandelwal N: Operative nuances to safeguard anomalous vertebral artery without compromising the surgery for congenital atlantoaxial dislocation: untying a tough knot between vessel and bone. $\mathbf{J}$ Neurosurg Spine 20:5-10, 2014

16. Salunke P, Futane S, Sharma M, Sahoo S, Kovilapu U, Khandelwal NK: 'Pseudofacets' or 'supernumerary facets' in congenital atlanto-axial dislocation: boon or bane? Eur Spine J 24:80-87, 2015

17. Salunke P, Futane S, Vaiphei K: Posterior bilateral supernumerary atlantoaxial facets: true or false joint? Spine (Phila Pa 1976) 38:E1633-E1635, 2013

18. Salunke P, Sahoo S, Deepak AN: Anomalous vertebral artery is not a deterrent to $\mathrm{C} 1-2$ joint dissection and manipulation for congenital atlantoaxial dislocation. Neurol India 63:1009-1012, 2015

19. Salunke P, Sahoo S, Khandelwal NK, Ghuman MS: Technique for direct posterior reduction in irreducible atlantoaxial dislocation: multi-planar realignment of C1-2. Clin Neurol Neurosurg 131:47-53, 2015

20. Salunke P, Sahoo SK, Deepak AN, Ghuman MS, Khandelwal $\mathrm{NK}$ : Comprehensive drilling of the $\mathrm{C} 1-2$ facets to achieve direct posterior reduction in irreducible atlantoaxial dislocation. J Neurosurg Spine 23:294-302, 2015

21. Salunke P, Sahoo SK, Futane S, Deepak AN, Khandelwal NK: 'Atlas shrugged': congenital lateral angular irreducible atlantoaxial dislocation: a case series of complex variant and its management. Eur Spine J 25:1098-1108, 2016

22. Salunke P, Sahoo SK, Ghuman MS: Bilateral inverted vertebral arteries (V3 segment) in a case of congenital atlantoaxial dislocation: Distinct entity or a lateral variant of persistent first intersegmental artery? Surg Neurol Int 5:82, 2014

23. Salunke P, Sharma M, Sodhi HB, Mukherjee KK, Khandelwal NK: Congenital atlantoaxial dislocation: a dynamic process and role of facets in irreducibility. $\mathbf{J}$ Neurosurg Spine 15:678-685, 2011

\section{Disclosures}

The authors report no conflict of interest concerning the materials or methods used in this study or the findings specified in this paper.

\section{Author Contributions}

Conception and design: Salunke. Acquisition of data: all authors. Analysis and interpretation of data: Salunke. Drafting the article: Salunke, Deepak. Critically revising the article: Salunke. Reviewed submitted version of the manuscript: Salunke. Approved the final version of the manuscript on behalf of all authors: Salunke. Statistical analysis: Deepak. Study supervision: Salunke.

\section{Correspondence}

Pravin Salunke, Department of Neurosurgery, PGIMER, Sector 12, Chandigarh 160012, India. email: drpravin_salunke@yahoo. co.uk. 